\title{
Novedoso algoritmo para la estimación de la inductancia de magnetización y la capacitancia del devanado de cada fase del transformador
}

ELECTRICAL AND ELECTRONICS ENGINEERING

\section{Novel algorithm for estimating the magnetization inductance and the winding capacitance of each phase of transformer}

\author{
Wilder Herrera ${ }^{\S *}$, Guillermo Aponte*, Eduardo Gómez*, Carlos Gonzalez-Garcia** \\ *Escuela de Ingenieria Electrica y Electronica, Universidad del Valle, Cali, Colombia \\ **Universidad Carlos III, Madrid, España \\ §wilder.herrera@correounivalle.edu.co guillermo.aponte@correounivalle.edu.co, \\ eduardo.gomez@correounivalle.edu.co,cagonzal@ing.uc3m.es
}

(Recibido: Mayo 25 de 2012-Aceptado: Abril 12 de 2013)

\begin{abstract}
Resumen
Este artículo describe un algoritmo novedoso para la estimación de la inductancia de magnetización y la capacitancia del devanado de cada fase del transformador, a partir de los datos de la curva de respuesta en frecuencia, utilizando un modelo del núcleo del transformador. El uso del modelo aplicado a las tres medidas de cada fase del transformador permite localizar y cuantificar una posible falla en baja frecuencia sin necesidad de disponer de las medidas FRA de referencia. Se hace la descripción del modelo, del algoritmo para obtener los parámetros de cada fase y de su aplicación en la interpretación de las variaciones en bajas frecuencias de la curva FRA, finalmente se muestran dos casos de aplicación.
\end{abstract}

Palabras clave: FRA, magnetización, modelado, principio de dualidad, transformador.

\section{Review}

This paper describes a novel algorithm for estimating the magnetization inductance and winding capacitance of each phase of transformer from the data of frequency response curve, using a transformer core model. The use of the model applied to the three measures for each phase of the transformer allows locating and quantifying a possible failure at low frequencies without need for reference FRA measures. It describes the model, the algorithm to obtain the parameters of each phase and its application in the interpretation of low-frequency variations of curve FRA, finally show two cases of application.

Keywords: Duality principle, FRA, magnetization, modeling, transformer. 


\section{Introducción}

El análisis de respuesta en frecuencia FRA (Frequency Response Analisys) es una herramienta para la evaluación de la condición de los transformadores, que permite detectar problemas como la deformación o el desplazamiento de los devanados. En el FRA se obtiene la impedancia o la función de transferencia del transformador, mediante la excitación con una señal periódica sinusoidal en un amplio rango de frecuencias. La amplitud y fase de las señales obtenidas de los terminales seleccionados de los transformadores, se representan directamente en función de la frecuencia, Dick \& Erven (1978).

En la actualidad el diagnóstico a partir de la técnica FRA se hace a través de la comparación entre dos curvas, obtenidas en tiempos de vida diferentes del transformador. Una curva representa el transformador en estado normal, mientras que la segunda curva representa el transformador en un supuesto estado de falla, las diferencias encontradas pueden indicar algún tipo de daño en el transformador. A pesar de que la técnica FRA ha mostrado validez para detectar si existe una falla o no, la interpretación física de la naturaleza de la falla es una tarea sumamente complicada, por lo que se presentan dificultades para ofrecer un diagnostico cualitativo (localización exacta de la falla) y cuantitativo (nivel del grado de falla), González \& Pleite (2010).

Para solventar esta dificultad distintos trabajos de investigaciónAbeywickrama, etal(2008), Mitchell \& Welsh (2011), Shintemirov et al (2010), Ang et al (2008) y organismos internacionales como IEC encargados de la elaboración de estándares para la aplicación de la técnica FRA (IEC 2012), apuestan por el uso de modelos equivalentes. Tanto en la comparación gráfica como la comparación basada en modelos, se hace necesario disponer de las medidas de referencia con las cuales se realiza el análisis comparativo. Sin embargo es posible detectar una falla comparando entre sí las curvas obtenidas de las tres fases del transformador trifásico, sin necesidad de disponer de curvas de referencia.
El presente trabajo describe un algoritmo novedoso basado en el uso de un modelo y la interpretación de las curvas FRA en el rango de baja frecuencia, que permite localizar y cuantificar una posible falla asociada al circuito magnético sin necesidad de disponer de las medidas FRA de referencia. En el punto II se describe el circuito del núcleo magnético del transformador y los fundamentos de la estructura del circuito equivalente que constituye el modelo.

\section{Circuito magnetico del núcleo del transformador}

Por lo general los transformadores se construyen sobre núcleos trifásicos de tres columnas, con cada fase colocada en una columna. En un transformador en condición normal la longitud del circuito magnético es igual para las dos fases laterales, mientras que la del circuito magnético de la fase central es menor, debido a que este circuito tiene menor reluctancia.

La asimetría entre los circuitos magnéticos de cada fase, se refleja en las curvas de respuesta en frecuencia. Cuando el transformador es excitado a través de una fase lateral, aparecen dos picos de resonancia, sin embargo cuando el transformador es excitado a través de la fase central aparece un solo pico de resonancia, esto se muestra en la Figura 1. Lo anterior se debe al recorrido del flujo en el núcleo, la fase central tiene dos caminos de igual reluctancia, en cambio la fase exterior tiene dos caminos de diferente reluctancia.

Una curva de respuesta en frecuencia típica del transformador, cuando es excitado a través de una fase lateral en el rango de bajas frecuencias, se muestra en la Figura 2, la cual ha sido dividida en tres rangos (Rango 1, Rango 2 y Rango $3)$. En el rango 1 que corresponde a muy baja frecuencia, la capacitancia del transformador puede ser despreciada y la respuesta es puramente inductiva, en este rango predomina la inductancia del circuito magnético. En el rango 2 los efectos inductivo y capacitivo empiezan a igualarse $\mathrm{y}$ 
aparecen los dos picos de resonancia típicos de las fases laterales, el máximo valor de cada pico de resonancia está definido principalmente por las pérdidas de potencia activa en el núcleo. En el rango 3 los efectos inductivos son despreciables y predomina el efecto capacitivo asociado con el circuito magnético. A más alta frecuencia el efecto del núcleo es despreciable.

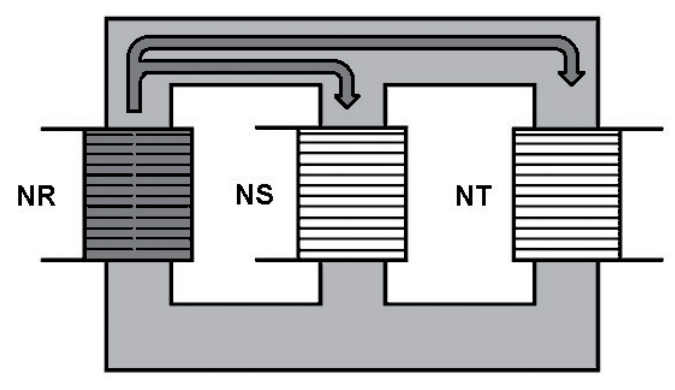

Fase Lateral

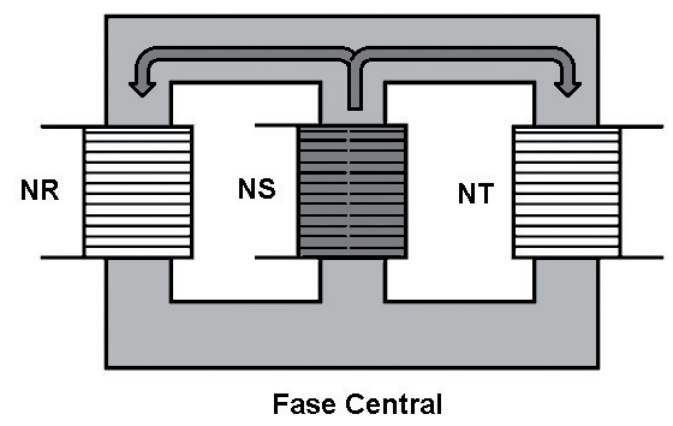

Fase Central

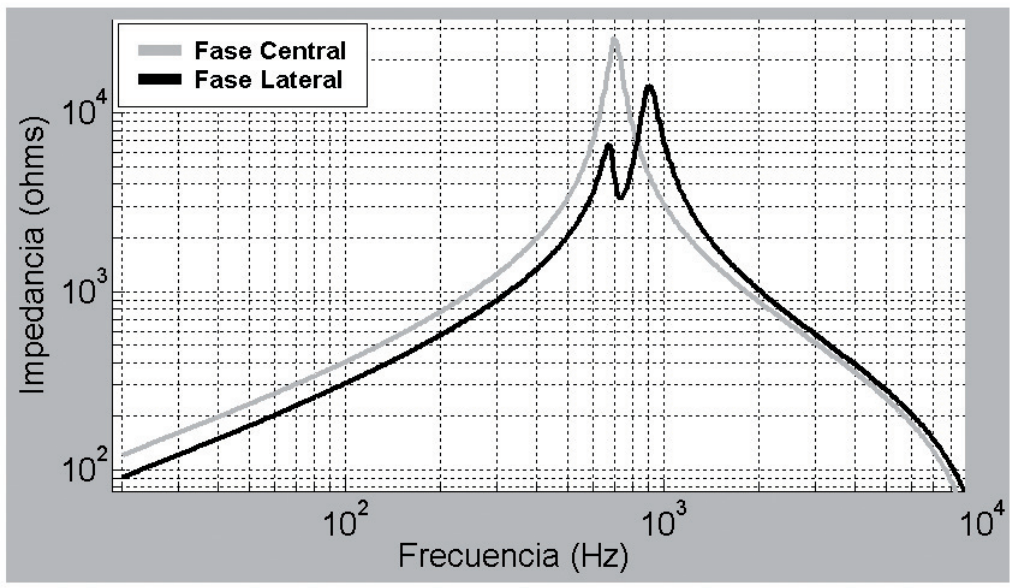

Figura 1. Diferencia entre las respuestas de la fase central y la fase lateral del transformador

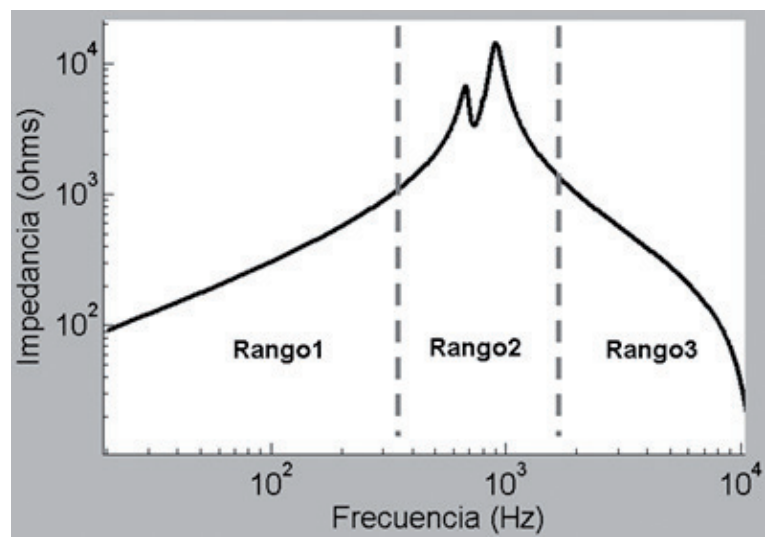

Figura 2. Respuesta en frecuencia de la fase lateral dividida en rangos
El circuito magnético equivalente del transformador se muestra en la Figura 3, donde se puede observar los 3 flujos magnéticos $\phi_{R^{\prime}}$ $\phi_{S} y \phi_{T}$ de las respectivas fases; aquí $\mathfrak{R}_{R}, \mathfrak{R}_{S} y \mathfrak{R}_{T}$ representan las reluctancias de los tres circuitos magnéticos; $f_{m R}, f_{m s} y f_{m T}$, son las fuerzas magnetomotrices presentes en cada fase y $f_{\mathfrak{R} R}, f_{\mathfrak{K} S}$, $f_{\mathfrak{k} T}$ son las fuerzas magneto-motrices que aparecen en cada reluctancia del circuito. La relación de las fuerzas magneto-motrices en cada malla del circuito magnético se presenta en Ec. (1) y (2); en Ec. (3) se muestra la sumatoria de los flujos de cada fase.

$$
\text { Malla 1: }-f_{m R}+f_{m S}+f_{\mathfrak{k} R}-f_{\mathfrak{x} S}=0
$$


Malla 2: $-f_{m S}+f_{m T}+f_{\mathfrak{k} S S}-f_{\mathfrak{k} T}=0$

$$
\Phi_{\mathrm{R}}+\Phi_{\mathrm{S}}+\Phi_{\mathrm{T}}=0
$$

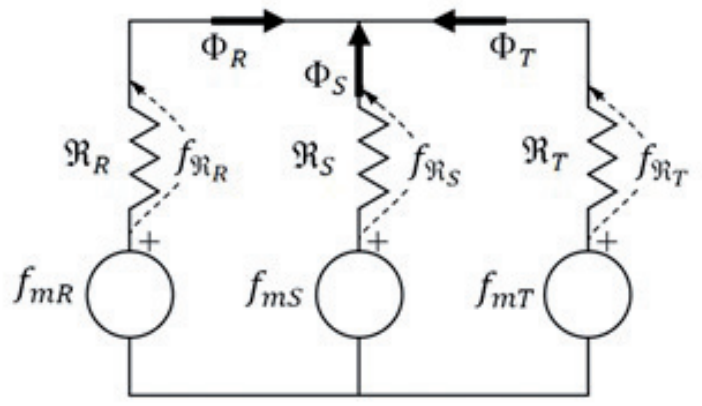

A)

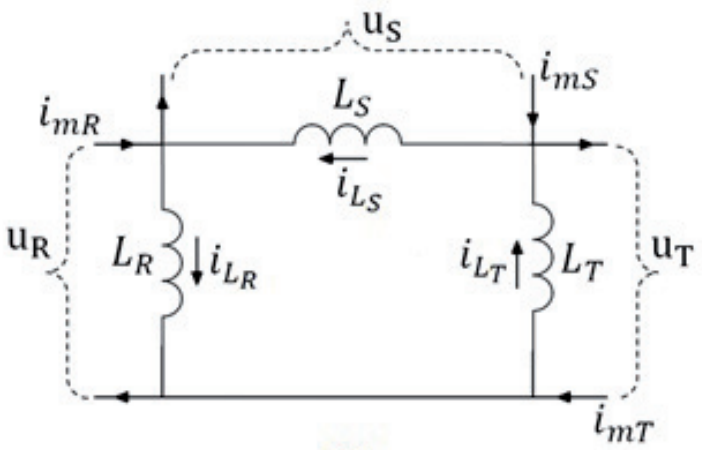

B)

Figura 3. A) Circuito magnético del núcleo del transformador. B) circuito eléctrico del transformador

Cada fuerza magneto-motriz del circuito magnético es producida por una componente de corriente, por lo que $i_{m R}, i_{m S}$ e $i_{m T}$, son las corrientes asociadas a las fuerzas magnetomotrices $f_{m R}, f_{m S} y$ $f_{m T}$. Del mismo modo $i_{L R}, i_{L S} e i_{L T}$ son las corrientes asociadas a las fuerzas magneto-motrices $f_{k R}$, $f_{\mathfrak{k} S}$ y $f_{\mathfrak{k} T}$. La relación entre las componentes de corrientes se muestra en Ec. (4) y (5); en Ec. (6) se muestra la relación de las correspondientes tensiones inducidas denominadas como $\mathrm{U}_{\mathrm{R}}, \mathrm{U}_{\mathrm{S}} \mathrm{y}$ $\mathrm{U}_{\mathrm{T}}$.

$$
\begin{gathered}
\text { Nodo 1: }-i_{m R}+i_{m S}+i_{L R}-i_{L S}=0 \\
\text { Nodo } 2:-i_{m S}+i_{m T}+i_{L S}-i_{L T}=0 \\
\mathrm{u}_{\mathrm{R}}+\mathrm{u}_{\mathrm{S}}+\mathrm{u}_{\mathrm{T}}=0
\end{gathered}
$$

Las ecuaciones Ec. (4), (5) y (6) describen el circuito eléctrico mostrado en la Figura 4, el cual es el dual del circuito magnético del núcleo del transformador y determina como se encuentran conectadas las inductancias de magnetización $\boldsymbol{L}_{R}, \boldsymbol{L}_{S}$ y $\boldsymbol{L}_{T}$, asociadas a cada fase del circuito magnético.

El modelo del núcleo del transformador se muestra en la Figura 4, Pleite et al. (2006), Gonzalez Garcia (2012), se construye a partir del circuito eléctrico de la Figura 3, adicionando los parámetros $R$ correspondientes a las pérdidas $\mathrm{y}$ $C$ que representan las capacitancias presentes en cada fase del transformador. El modelo consta de tres celdas conectadas y los parámetros de cada celda representan las características de cada fase. Los parámetros $L_{R}, L_{S} y L_{T}$, que representan las inductancias de magnetización de cada fase del transformador, se relacionan con las reluctancias de la fase, como se indica en Ec. (7).

$$
L_{R}=\frac{N_{R}^{2}}{\Re_{R}} \quad L_{S}=\frac{N_{S}{ }^{2}}{\Re_{S}} \quad L_{T}=\frac{N_{T}{ }^{2}}{\Re_{T}}
$$

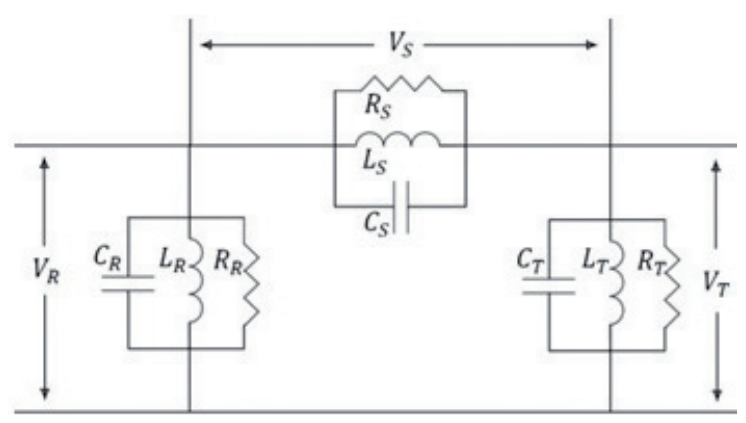

A)

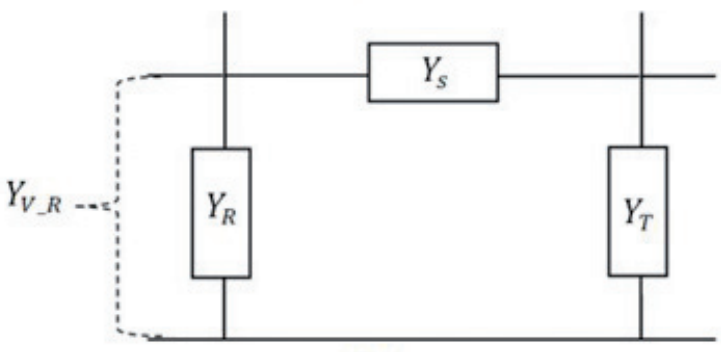

B)

Figura 4. A) Modelo del núcleo del transformador. B) Modelo representado por tres admitancias $Y_{R}, Y_{S}$ y $Y_{T}$ que contienen características propias de cada fase 
En un transformador en estado sano la inductancia $L_{S}$ será mayor que las inductancias laterales, debido a que el circuito magnético de la fase central tiene menor reluctancia. Por otro lado las inductancias $L_{R}$ y $L_{T}$ serán aproximadamente iguales.

Los parámetros $R_{R}, R_{S} y R_{T}$, representan las pérdidas de potencia activa en cada fase del núcleo trifásico. En Ec. (8) se muestra una relación entre la pérdida de potencia activa y el valor del parámetro resistivo para cada fase del transformador.

$$
R_{R}=\frac{V_{R}^{2}}{\text { Watt }_{R}} \quad R_{S}=\frac{V_{S}^{2}}{\text { Watt }_{S}} \quad R_{T}=\frac{V_{T}^{2}}{\text { Watt }_{T}}
$$

En un transformador en estado sano el valor de $R_{S}$ será mayor que $R_{R}$ y $R_{T}$, debido a que el circuito magnético de la fase central tiene menor longitud y por lo tanto menores perdidas, además $R_{R}$ y $R_{T}$ deben ser aproximadamente iguales.

Por último los parámetros $C_{R}, C_{S} y C_{T}$, representan la capacitancia del devanado de cada fase, que tiene influencia en el rango de baja frecuencia. En un transformador en estado sano estos parámetros son aproximadamente iguales, debido a que los devanados de las tres fases son construidos con la misma geometría.

$$
C_{R}=C_{S}=C_{T}
$$

El comportamiento del transformador en baja frecuencia es no lineal, fundamentalmente debido al comportamiento del núcleo ferromagnético el cual presenta un ciclo de histéresis. Sin embargo ya que el tipo de medidas FRA son de pequeña señal (típicamente $10 \mathrm{Vpp}$ ), la saturación del núcleo no tiene influencia en la respuesta, por lo que el modelo obtenido es apropiado para estudios del transformador en condiciones de pequeña señal. Para tipos de medida de mayor señal, la permeabilidad del núcleo cambia y por lo tanto cambia la inductancia de magnetización.

\section{Algoritmo de estimación de parámetros del modelo}

Para estimar los parámetros del modelo se deben tener las tres curvas de impedancia en frecuencia, medidas en configuración de circuito abierto desde cada fase del transformador. La curva de impedancia en frecuencia es un número complejo, que cobra determinado valor para cada frecuencia en un ancho de banda. La técnica FRA es una prueba que se realiza por fase, debido a que las tres fases del transformador están acopladas magnéticamente, la curva medida desde una fase contiene información mezclada de los circuitos magnéticos de las tres fases. La curva de impedancia en frecuencia medida obtenida desde la fase $\mathrm{R}$ se denomina $Z_{M_{-},}$y las medidas desde las fases $\mathrm{S}$ y $\mathrm{T}$ como $Z_{M_{-} S} y Z_{M_{-} T}$.

A partir de las curvas de impedancia medida $Z_{M}$, se pueden obtener las curvas de admitancias medidas desde las diferentes fases $Y_{M R}, Y_{M S} y Y_{M T}$ , simplemente como el inverso de la impedancia. La curva de admitancia en frecuencia medida desde una fase, contiene también información mezclada de las fases restantes del transformador, por lo que una variación en el circuito magnético de alguna de las tres fases se verá reflejada en las tres curva medidas.

El modelo del núcleo del transformador se puede considerar como tres admitancias $Y_{R}, Y_{S}$ y $Y_{T}$ conectadas como se muestra en la Figura 4. En donde $Y_{R}$, es una admitancia que contiene características propias de la fase $\mathrm{R} ; Y_{S}$ y $Y_{T}$ son las admitancias propias de las fases $\mathrm{S}$ y $\mathrm{T}$. Las admitancias del modelo, vistas desde sus respectivas fases son iguales a:

$$
\begin{aligned}
& Y_{V_{-} R}\left(\omega_{i}\right)=Y_{R}\left(\omega_{i}\right)+\left(\frac{Y_{s}\left(\omega_{i}\right) \cdot Y_{T}\left(\omega_{i}\right)}{Y_{S}\left(\omega_{i}\right)+Y_{T}\left(\omega_{i}\right)}\right) \\
& Y_{V_{-} S}\left(\omega_{i}\right)=Y_{S}\left(\omega_{i}\right)+\left(\frac{Y_{R}\left(\omega_{i}\right) \cdot Y_{T}\left(\omega_{i}\right)}{Y_{R}\left(\omega_{i}\right)+Y_{T}\left(\omega_{i}\right)}\right) \\
& Y_{V_{-} T}\left(\omega_{i}\right)=Y_{T}\left(\omega_{i}\right)+\left(\frac{Y_{S}\left(\omega_{i}\right) \cdot Y_{R}\left(\omega_{i}\right)}{Y_{R}\left(\omega_{i}\right)+Y_{T}\left(\omega_{i}\right)}\right)
\end{aligned}
$$


Donde:

$$
\begin{gathered}
Y_{R}\left(\omega_{i}\right)=\frac{1}{R_{R}}+j\left(\omega_{i} C_{R}-\frac{1}{\omega_{i} L_{R}}\right) \\
Y_{S}\left(\omega_{i}\right)=\frac{1}{R_{S}}+j\left(\omega_{i} C_{S}-\frac{1}{\omega_{i} L_{S}}\right) \\
Y_{T}\left(\omega_{i}\right)=\frac{1}{R_{T}}+j\left(\omega_{i} C_{T}-\frac{1}{\omega_{i} L_{T}}\right)
\end{gathered}
$$

Durante el procedimiento iterativo se calculan las admitancias de referencia para cada fase, a partir de la cual se estiman los parámetros del modelo. Para el caso de la fase R la admitancia de referencia se obtiene a partir de Ec. (10), en este caso si despejamos la admitancia propia de la fase R tenemos:

$$
\begin{aligned}
& Y_{R}\left(\omega_{i}\right)=Y_{V_{-} R}\left(\omega_{i}\right)-\left(\frac{Y_{s}\left(\omega_{i}\right) \cdot Y_{T}\left(\omega_{i}\right)}{Y_{S}\left(\omega_{i}\right)+Y_{T}\left(\omega_{i}\right)}\right) \\
& Y_{r \varepsilon f_{-} R}=Y_{M_{-} R}\left(\omega_{i}\right)-\left(\frac{Y_{S}\left(\omega_{i}\right) \cdot Y_{T}\left(\omega_{i}\right)}{Y_{S}\left(\omega_{i}\right)+Y_{T}\left(\omega_{i}\right)}\right)=G_{r e f}\left(\omega_{i}\right)+j \cdot B_{r \theta f}\left(\omega_{i}\right) \\
& R=\frac{m}{\sum_{i=1}^{m} G_{r e f}\left(\omega_{i}\right)} \\
& L=\frac{\sum_{i=1}^{m} \omega_{i}{ }^{2} \cdot \sum_{i=1}^{m} \frac{1}{\omega_{i}^{2}}-m^{2}}{m \cdot \sum_{i=1}^{m} \omega_{i} B_{r e f}\left(\omega_{i}\right)-\sum_{i=1}^{m} \frac{B_{r e f}\left(\omega_{i}\right)}{\omega_{i}} \cdot \sum_{i=1}^{m} \omega_{i}{ }^{2}} \\
& C=\frac{\sum_{i=1}^{m} \omega_{i} B_{r \theta f}\left(\omega_{i}\right) \cdot \sum_{i=1}^{m} \frac{1}{\omega_{i}^{2}}-m \cdot \sum_{i=1}^{m} \frac{B_{r \varepsilon f}\left(\omega_{i}\right)}{\omega_{i}}}{\sum_{i=1}^{m} \omega_{i}{ }^{2} \cdot \sum_{i=1}^{m} \frac{1}{\omega_{i}^{2}}-m^{2}} \\
& E(G, B, C)=\frac{1}{m} \sum_{i=1}^{m}\left|Y_{r e f}\left(\omega_{i}\right)-\frac{1}{R}+j \cdot\left(\omega_{i} C-\frac{1}{\omega_{i} L}\right)\right|^{2}
\end{aligned}
$$

La admitancia de referencia de la fase $\mathrm{R}$ es una aproximación de la admitancia propia, y esta definida como la admitancia medida $Y_{M R}(\omega i)$, descontando el efecto de las admitancias propias de las fases restantes como se muestra en Ec. (17).

Los parámetros de cada fase del modelo se obtienen a partir de los datos de la admitancia de referencia, empleando las siguientes ecuaciones, Pleite et al. (2002), Guillermo et al. (2011), Guillermo et al. (2010).

Donde $m$ es el número de puntos incluidos en el ancho de banda asignada. $Y_{\text {ref }}(\omega i)$ es la admitancia de referencia; $G_{r e f}(\omega i)$ y $\left.B_{r e f} \omega i\right)$ son la conductancia y susceptancia de la admitancia de referencia en cada punto y ( $\omega$ i) es la frecuencia angular. Las anteriores ecuaciones resultan de la minimización del error definido, tal

\section{Procedimiento Iterativo}

El procedimiento iterativo comienza en la fase central, los parámetros de cada fase $R, L$ y $C$ deben ser inicializados. La inicialización correcta de los valores de los parámetros, es importante porque ayuda a que el algoritmo converja rápidamente a la solución, sin embargo por simplicidad la inicialización del algoritmo de cálculo puede ser determinada al definir los valores de los parámetros del modelo iguales a cero. Para cada fase se calcula una admitancia de referencia $Y_{\text {ref }}$, la cual se utiliza para calcular los parámetros. 
Paso1. Calculo de las admitancias de cada fase Calculo de los parámetros de la fase $S$ :

Se calcula la admitancia $Y_{\text {ref_S }}(\omega i)$ de la respuesta de la fase S, como la admitancia medida $Y_{M_{S} S}(\omega i)$ descontando el efecto del paralelo de las admitancias propias de las fases R y T, como:

$$
Y_{r e f_{-} S}=Y_{M_{-} S}\left(\omega_{i}\right)-\left(\frac{Y_{R}\left(\omega_{i}\right) \cdot Y_{T}\left(\omega_{i}\right)}{Y_{R}\left(\omega_{i}\right)+Y_{T}\left(\omega_{i}\right)}\right)
$$

A través de la admitancia de referencia se obtienen los parámetros $R_{S}, C_{S} y L_{S}$, utilizando las ecuaciones (18), (19) y (20). Se calcula la admitancia propia de la fase $\mathrm{S}$, como:

$$
Y_{S}=\left(\frac{1}{R_{S}}\right)+j \omega_{i} C_{S}+\frac{1}{j \omega_{i} L_{S}}
$$

Calculo de los parámetros de la fase $R$ :

Se calcula la admitancia $Y_{\text {ref } R}(\omega i)$ de la respuesta de la fase $\mathrm{R}$, como la admitancia medida $Y_{M_{-} R}(\omega i)$ descontando el efecto del paralelo de las admitancias propias de las fases $\mathrm{S}$ (calculada en el paso anterior) y la admitancia de la fase $\mathrm{T}$ como:

$Y_{r f_{-} R}=Y_{M_{-} R}\left(\omega_{i}\right)-\left(\frac{Y_{S}\left(\omega_{i}\right) \cdot Y_{T}\left(\omega_{i}\right)}{Y_{S}\left(\omega_{i}\right)+Y_{T}\left(\omega_{i}\right)}\right)$

A través de la admitancia de referencia se obtienen los parámetros $R_{R}, C_{R} y L_{R}$, utilizando las ecuaciones Ec. (18), (19) y (20). Se calcula la admitancia propia de la fase $\mathrm{R}$, como:

$$
Y_{R}=\left(\frac{1}{R_{R}}\right)+j \omega_{i} C_{R}+\frac{1}{j \omega_{i} L_{R}}
$$

\section{Calculo de los parámetros de la fase T:}

Se calcula la admitancia $Y_{\text {ref_T }}(\omega i)$ de la respuesta de la fase $\mathrm{T}$, como la admitancia medida $Y_{M_{-} T}(\omega i)$ descontando el efecto del paralelo de las admitancias propias de las fases $\mathrm{R}$ y $\mathrm{S}$, calculadas en el paso anterior, como:

$$
Y_{r \in f_{-} T}=Y_{M_{-} T}\left(\omega_{i}\right)-\left(\frac{Y_{R}\left(\omega_{i}\right) \cdot Y_{S}\left(\omega_{i}\right)}{Y_{R}\left(\omega_{i}\right)+Y_{S}\left(\omega_{i}\right)}\right)
$$

A través de la admitancia de referencia se obtienen los parámetros $R_{T}, C_{T}$ y $L_{T}$, utilizando las ecuaciones Ec. (18), (19) y (20). Se calcula la admitancia propia de la fase $\mathrm{T}$, como:

$$
Y_{T}=\left(\frac{1}{R_{T}}\right)+j \omega C_{T}+\frac{1}{j \omega L_{T}}
$$

Paso 2. Cálculo del error relativo promedio ERP

Se calcula la admitancia del modelo vista desde cada fase utilizando las ecuaciones Ec. (10), (11) y (12). El error relativo promedio ERP En cada fase es igual a:

$$
\begin{aligned}
& E R P_{-} R=\left(\frac{1}{m}\right) \cdot \sum_{i=1}^{m} \frac{\left|Y_{M_{-} R}\left(\omega_{i}\right)-Y_{V_{-} R}\left(\omega_{i}\right)\right|}{\left|Y_{M_{-} R}\left(\omega_{i}\right)\right|} \\
& E R P_{-} S=\left(\frac{1}{m}\right) \cdot \sum_{i=1}^{m} \frac{\left|Y_{M_{-} S}\left(\omega_{i}\right)-Y_{V_{-} S}\left(\omega_{i}\right)\right|}{\left|Y_{M_{-} S}\left(\omega_{i}\right)\right|} \\
& E R P_{-} T=\left(\frac{1}{m}\right) \cdot \sum_{i=1}^{m} \frac{\left|Y_{M_{-} T}\left(\omega_{i}\right)-Y_{V_{-} T}\left(\omega_{i}\right)\right|}{\left|Y_{M_{-} T}\left(\omega_{i}\right)\right|}
\end{aligned}
$$

\section{Paso 3. Evaluación del error:}

Se evalúan los errores de cada fase, de la respuesta del modelo respecto a la respuesta medida. Si los errores no son suficientemente bajos, los parámetros de cada fase son recalculados retornando de nuevo al paso1. En la Figura 5. se muestra un diagrama de bloques del algoritmo.

\section{Resultados y discusión}

\section{A. Caso 1: Transformador en estado normal}

A continuación se presenta la aplicación del algoritmo en un transformador en buen estado de 90 MVA, $115 / 13.2 \mathrm{kV}$, DY, con núcleo de tres columnas. Las curvas de referencia medidas de las tres fases R, S y T del lado de baja, se muestran en la Figura 6. Al aplicar el algoritmo para el Caso 
1, se estiman las inductancias de magnetización y las capacitancias del devanado de cada fase del transformador, los valores de estos parámetros se muestran en la Tabla I.

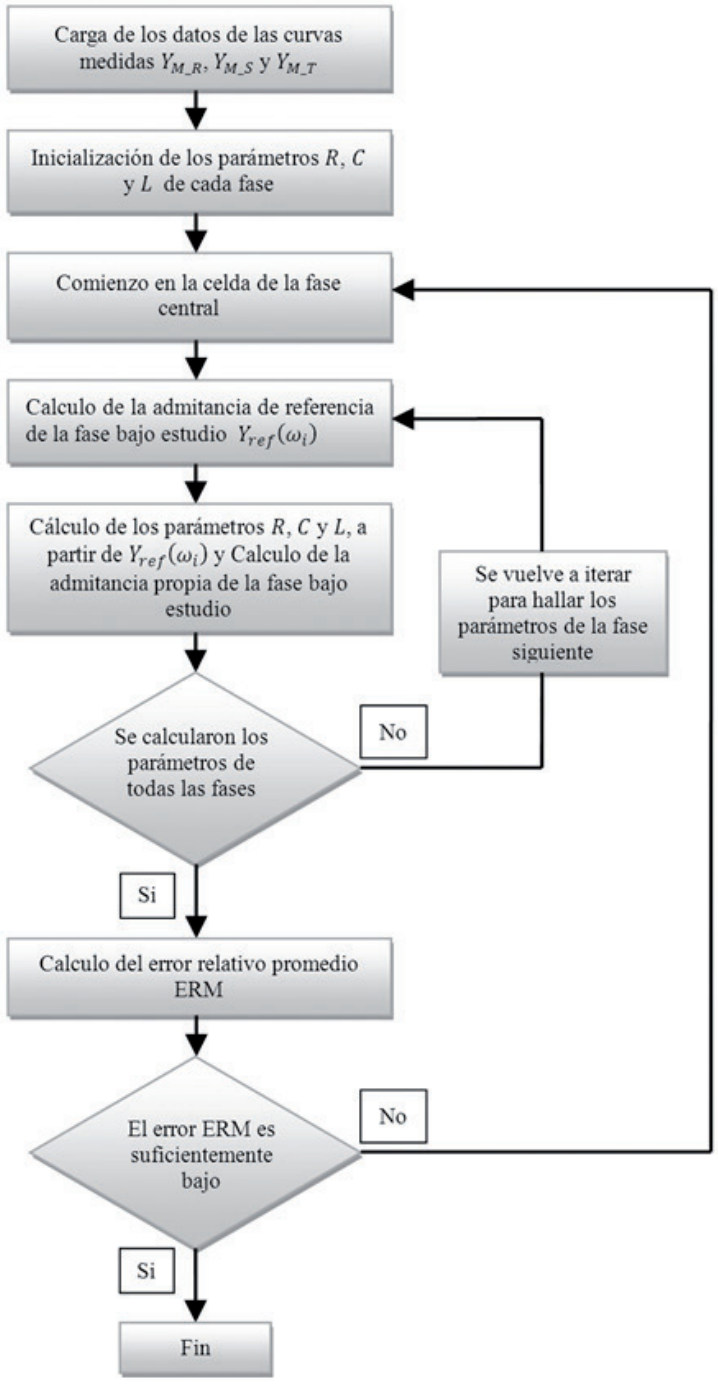

Figura 5. Diagrama de bloques del algoritmo

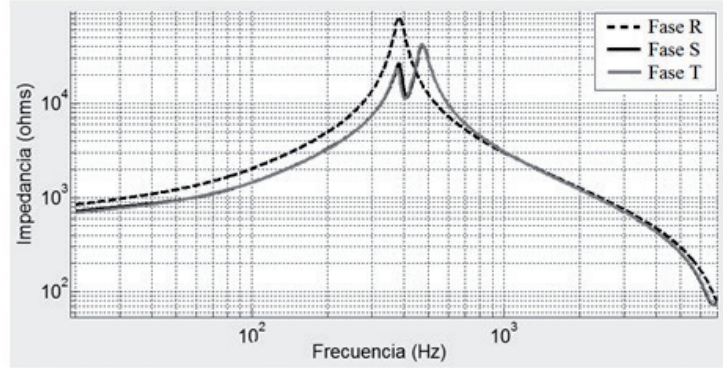

A)

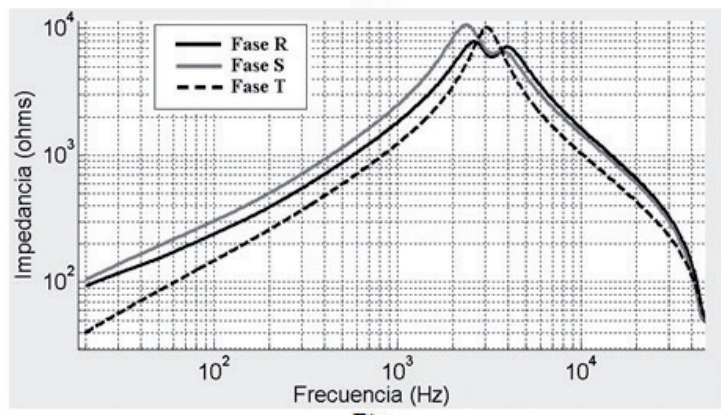

B)

Figura 6. Curvas de respuesta en frecuencia medidas en las tres fases del transformador. A) Caso I. B) Caso II.

De la Tabla I se observa que la inductancia $L_{S}$ de la fase central es mayor que las inductancias $L_{R} \mathrm{y}$ $L_{T}$, como era de esperarse ya que la reluctancia de la fase central del transformador es menor que las reluctancias de las fases laterales. Por otro lado se observa que las inductancias $L_{R}$ y $L_{T}$, son aproximadamente iguales, con una diferencia del $1.87 \%$, debido a que la longitud de los circuitos magnéticos de las fases laterales son aproximadamente iguales. En el caso de las capacitancias, se observa que sus valores son aproximadamente iguales en las tres fases, y lo cual se debe a que los devanados de las tres fases han sido construidos con la misma geometría.

Tabla I. Valores de las inductancias y capacitancias de magnetización para el Caso 1

\begin{tabular}{cccccc}
\hline \multicolumn{5}{c}{ Valores de los parámetros } \\
\hline & Fase R & Fase S & Fase T \\
$R_{R}[\Omega]$ & 80117.25 & $R_{s}[\Omega]$ & 155270.85 & $R_{T}[\Omega]$ & 86352.21 \\
$L_{R}[H]$ & 2.669 & $L_{s}[H]$ & 6.225 & $L_{T}[H]$ & 2.620 \\
$C_{R}[F]$ & $4.291 \mathrm{e}-8$ & $C_{s}[F]$ & $3.873 \mathrm{e}-8$ & $C_{T}[F]$ & $4.330 \mathrm{e}-8$ \\
\hline
\end{tabular}




\section{B. Caso 2: Transformador en estado de falla}

Este caso corresponde a la aplicación del procedimiento en un transformador de $21 \mathrm{MVA}$, 34.5 / $13.8 \mathrm{kV}$, YY, con núcleo de tres columnas. El transformador presenta una falla de bloqueo en el mecanismo que desplaza los Taps de la

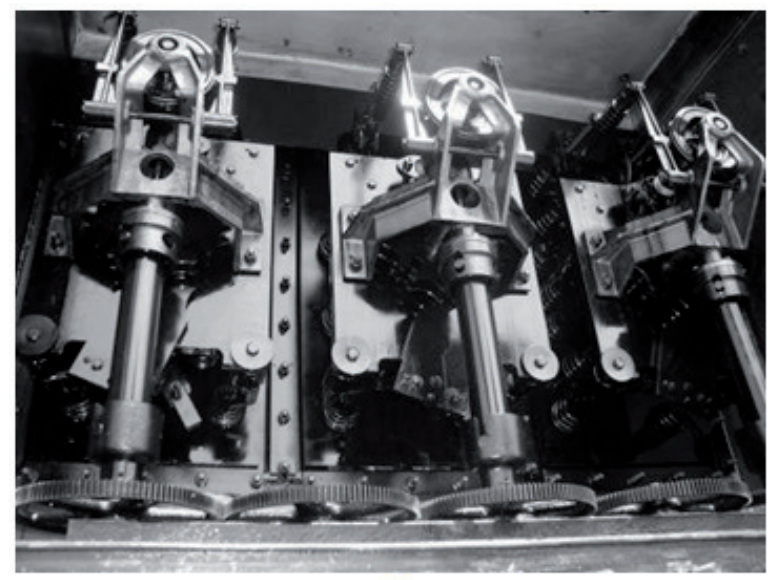

A)

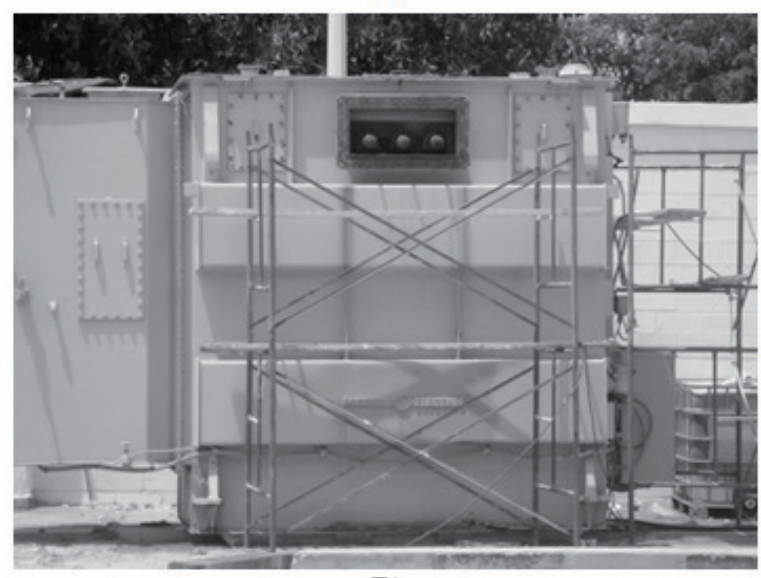

B)

Figura 7. A) Armario de accionamiento del cambiador de Taps LTC. B) Transformador bajo prueba fase T. Durante la prueba, el cambiador de Taps LTC se encontraba en la posición 33. Las curvas medidas de las tres fases R, S y T del lado de baja, se muestran en la Figura 6. La Figura 7 muestra el mecanismo de accionamiento del cambiador de Taps LTC y una vista del transformador bajo prueba.

Al aplicar el algoritmo para el Caso 2, se estiman las inductancias de magnetización y las capacitancias del devanado de cada fase del transformador, los valores de estos parámetros se muestran en la Tabla II. En un transformador en estado normal, la longitud de los circuitos magnéticos de las fases laterales son aproximadamente iguales, por lo que también deben serlo las inductancias $L_{T} y$ $L_{R}$. De la Tabla II se puede observar que el valor de la inductancia $L_{T}$ es menor al valor de $L_{R}$, el error es de $57.8 \%$ con respecto a la fase $\mathrm{T}$. Al estar bloqueado el mecanismo de desplazamiento de Taps de la fase $\mathrm{T}$, se disminuye el número de espiras de este devanado en comparación al devanado lateral de la fase $\mathrm{R}$, por lo que esto se refleja como una disminución de la inductancia de magnetización $L_{T}$ en comparación con su fase homologa opuesta $L_{R}$.

La disminución de espiras en la fase $\mathrm{T}$, aumenta también la capacitancia serie y por lo tanto hay una concentración de potencial eléctrico en el devanado, lo que se refleja como un aumento de la capacitancia $C_{T}$. De la Tabla II se observa que el valor de $C_{T}$ es mayor que las capacitancias de las otras fases. El error entre las capacitancias $C_{R}$ y $C_{S}$ es de $52.3 \%$ con respecto a la fase T. En contraste las capacitancias $C_{R}$ y $C_{S}$ tienen valores muy similares con un error de $9.9 \%$ con respecto a la fase $\mathrm{S}$.

Tabla 2. Valores de las inductancias y capacitancias de magnetización para el Caso 2

\begin{tabular}{cccccc}
\hline \multicolumn{5}{c}{ Valores de los parámetros } \\
\hline & Fase R & \multicolumn{5}{c}{ Fase S } & Fase T \\
$R_{R}[\Omega]$ & 17080.82 & $R_{s}[\Omega]$ & 24284.335 & $R_{T}[\Omega]$ & 13425.24 \\
$L_{R}[H]$ & 0.3057 & $L_{s}[H]$ & 1.0077 & $L_{T}[H]$ & 0.1937 \\
$C_{R}[F]$ & $6.064 \mathrm{e}-9$ & $C_{s}[F]$ & $6.731 \mathrm{e}-9$ & $C_{T}[F]$ & $1.272 \mathrm{e}-8$ \\
\hline
\end{tabular}




\section{Conclusiones}

Se ha presentado un algoritmo que permite estimar los parámetros de un modelo del núcleo del transformador, a partir de los datos de las curvas de respuesta en frecuencia.

El modelo puede utilizarse para evaluar las curvas FRA a bajas frecuencias, ya que los parámetros tienen significado físico de los fenómenos que ocurren en el núcleo trifásico.

El procedimiento de cálculo es capaz de estimar las inductancias y capacitancias de cada fase por separado, por lo que permite identificar en qué fase se encuentra la falla, sin necesidad de disponer de las medidas FRA de referencia.

\section{Agradecimientos}

Los autores agradecen a COLCIENCIAS en Colombia, por su apoyo en el desarrollo de este trabajo a través del programa de formación doctoral, convocatoria 494 de 2009.

\section{Referencias bibliográficas}

Abeywickrama, N., Serdyuk, Y.V. \& Gubanski, S.M. (2008). High-Frequency Modeling of Power Transformers for Use in Frequency Response Analysis (FRA). IEEE Transactions on Power Delivery, 23 (4), pp. 2042-2049.

Ang, S.P., Li, J., Wang, Z. \& Jarman, P. (2008). FRA low frequency characteristic study using duality transformer core modeling. In International Conference on Condition Monitoring and Diagnosis (CMD), Beijing, China, pp. 889-893.

Dick, E.P. \& Erven, C.C. (1978). Transformer Diagnostic Testing by Frequuency Response Analysis. IEEE Transactions on Power Apparatus and Systems, 97 (6), 2144-2153.

Gonzalez Garcia, C. (2012). Procedimiento de Modelado basado en el Análisis de la Respuesta en Frecuencia y aplicación en Transformadores
Trifásicos de Potencia para su Caracterización y Diagnóstico. Doctoral Thesis, Universidad Carlos III de Madrid, Madrid, Spain.

González, C. \& Pleite, J. (2010). Transformer modeling approaches for Frequency Response Analysis. In XIX International Conference on Electrical Machines (ICEM), Roma, Italy, 1-6.

Aponte, G., Cadavid, H., Martinez, J., Castaño, J. \& Herrera, W. (2010). Evaluación de la Respuesta en Frecuencia del Transformador utilizando un modelo Circuital de Celdas. Revista Energía y Computación, 18, 1-8.

Aponte, G., Herrera, W., Gonzalez, C. \& Pleite, J. (2011). Implementación de un Modelo de un Transformador Eléctrico para el Análisis de su Respuesta en Frecuencia. Información Tecnológica, 22 (4), 59-72.

IEC (International Electrotechnical Commission). (2012). Power transformers-Part 18: Measurement of frequency response. International Standard, IEC 60076-18.

Mitchell, S.D. \& Welsh, J.S. (2011). Modeling Power Transformers to Support the Interpretation of Frequency-Response Analysis. IEEE Transactions on Power Delivery, 26 (4), 27052717.

Pleite, J., Gonzalez, C., Vazquez, J. \& Lazaro, A. (2006). Power transformer core fault diagnosis using frequency response analysis. In IEEE Mediterranean Electrotechnical Conference (MELECON), Malaga, Spain, 1126-1129.

Pleite, J., Olias, E., Barrado, A., Lazaro, A. \& Vazquez, J. (2002). Transformer modeling for FRA techniques. In Transmission and Distribution Conference and Exhibition: Asia Pacific. IEEE/ PES, Yokohama, Japan, 317- 321.

Shintemirov, A., Tang, W.H. \& Wu, Q.H. (2010). Transformer Core Parameter Identification Using Frequency Response Analysis. IEEE Transactions on Magnetics, 46 (1), 141-149. 